Published in final edited form as:

Nature. 2012 September 6; 489(7414): 43-44. doi:10.1038/489043a.

\title{
A protein engagement RING
}

\author{
Christopher D. Lima and \\ Structural Biology Program, Sloan-Kettering Institute, New York, New York 10065, USA \\ Brenda A. Schulman \\ Howard Hughes Medical Institute, Department of Structural Biology, St. Jude Children's Research \\ Hospital, Memphis, Tennessee 38105, USA
}

\begin{abstract}
The mechanistic details of the attachment of a small protein, ubiquitin, to other proteins are unclear. Crystal structures of the E2-ubiquitin and RING E3 enzymes, engaged and ready for transfer, provide fresh insights.
\end{abstract}

Cells use molecular tags to modulate the fates and functions of proteins. One such tag is ubiquitin, a small protein that regulates nearly every facet of the life of eukaryotic cells (those found in organisms such as animals, plants and fungi). Tagging a protein with ubiquitin requires the sequential action of three types of enzymes: E1 activating enzymes attach ubiquitin to a cysteine residue on E2 conjugating enzymes, and E3 ligases stimulate ubiquitin transfer from E2-ubiquitin onto a lysine of the substrate protein. How E3 enzymes - the most common of which belong to the RING family 1 - carry out the final step has remained a longstanding mystery. Now Plechanovová et al. ${ }^{2}$ (on page 115 of this issue) and Dou et al..$^{3}$ (writing in Nature Structural \& Molecular Biology) illuminate such a mechanism at high resolution, by describing the structures of RING E3 ligases bound to E2-ubiquitin. Their results suggest a mode of action that could apply to other E3 enzymes that stimulate ubiquitin transfer.

More than 600 human genes encode RING or RING-like E3 ligases, underscoring their biological importance ${ }^{1}$. Canonical RING proteins contain a zinc-binding region that is rich in cysteine and histidine residues and that, on its own, can bind to E2-ubiquitin and promote ubiquitin transfer ${ }^{1}$. Previous crystal structures revealed some of the interactions between E2 and E3 enzymes, but none of them had captured the elusive association of an E2-ubiquitin intermediate and a RING E3 ligase. This was largely because the link between E2 and ubiquitin is a labile thioester bond.

Plechanovová et al. and Dou et al. cleverly overcame this challenge by using engineered E2 proteins that were linked to ubiquitin through more stable bond types (peptide and oxyester, respectively). Both groups of researchers mixed their engineered E2-ubiquitin with an E3 RING ligase (RNF4 or BIRC7) and then purified, and determined the crystal structures of, the resulting RING-E2-ubiquitin protein complexes. For the E3 ligase, Dou et al. used a dimeric BIRC7, whereas Plechanovová et al. used a tandem protein fusion (RNF4-RNF4) to mimic the RNF4 dimer.

Earlier studies showed that, in the absence of an E3 partner, E2-ubiquitin can adopt many inactive ('open') configurations ${ }^{4}$, which presumably prevent the transfer of the molecular ubiquitin tag to a substrate protein (Fig. 1a). The structures determined by Plechanovová et 
al. and Dou et al. reveal that RING E3 ligases lock E2-ubiquitin into an activated, closed conformation (Fig. 1b) that is poised for ubiquitin transfer; such a form has also been described in concurrent studies of similar proteins using nuclear magnetic resonance $\mathrm{e}^{3,5}$.

In the RING-E2-ubiquitin crystal structures, certain amino-acid residues of one of the two RING monomers interact with both ubiquitin and the E2 protein. Of note, an arginine side chain of a RING monomer bridges the E2 protein and the $\mathrm{C}$-terminal tail of ubiquitin. The opposite RING subunit also contacts ubiquitin through, for example, a highly-conserved tyrosine or phenylalanine residue. Moreover, a zinc-bound histidine (which is characteristically found in canonical RING proteins) interacts with ubiquitin through a hydrogen bond.

The crystal structures also show an extensive network of interactions between the E2 protein and its linked ubiquitin. In particular, Plechanovová et al. describe a hydrogen bond between a carbonyl oxygen of ubiquitin's C-terminal tail and a highly conserved asparagine side chain of the E2 protein; this asparagine is known ${ }^{6}$ to be required for efficient ubiquitin transfer. In addition, an aspartate residue of the E2 protein, which had previously been shown to have a role in activating the substrate protein's lysine ${ }^{7}$, is rearranged in the RINGE2-ubiquitin complexes.

The findings support a model by which RING binding reduces the conformational heterogeneity of E2-ubiquitin and constrains ubiquitin's C-terminal tail in a shallow cleft within the E2 protein. As a result, the thioester bond becomes suitably positioned for attack by the substrate protein's lysine, and several residues of the E2 protein are rearranged to promote the transfer reaction. Both groups of authors validated the model through careful biochemical studies. For example, ubiquitin transfer was abolished by making amino-acid changes in the E3 ligase that were predicted to impair its interactions with ubiquitin or the E2 (ref 8). Moreover, Plechanovová and colleagues describe that their E2-ubiquitin is a competitive inhibitor of E3-mediated ubiquitin transfer to substrate proteins; this result confirms that E2-ubiquitin (in which the two proteins are linked through a peptide bond instead of a thioester) is structurally similar to natural E2-ubiquitin.

Does the model hold for other E2 and E3 proteins? An earlier study showed that the nonRING E3 ligase (RanBP2) enhances conjugation by coordinating E2-SUMO optimally for substrate binding and catalysis ${ }^{9}$ (SUMO is a ubiquitin-like protein) and the RING-E2ubiquitin structures show striking similarities to the protein complex formed by the nonRING E3 ligase (RanBP2), an E2 protein and a SUMO-tagged protein substrate ${ }^{9}$ (Fig. 1c). Furthermore, Plechanovová et al. show that CHIP, an E3 ligase belonging to the RING-like U-box family, also stimulates ubiquitin transfer by rearranging E2-ubiquitin into a closed configuration and computer modeling ${ }^{10,11}$ and NMR data ${ }^{5}$ have indicated that some monomeric RING, or RING-related (SP-RING), E3 ligases contain elements that could lock E2-ubiquitin or E2-SUMO into a closed conformation.

However, there is evidence that, for some E2 proteins, E2-ubiquitin can adopt a closed configuration in the absence of E3 ligases ${ }^{12-14}$. And it is unclear whether some other types of E3 ligases, which transfer ubiquitin through mechanisms different from those used by RING proteins, will follow the model described by the authors. For example, for E3 ligases of the HECT and RBR families, ubiquitin is transferred from an E2 protein onto a cysteine on the E3 enzyme, before being attached to the protein substrate. Although details of the second step await elucidation, it has been reported ${ }^{15}$ that HECT binding to E2-ubiquitin promotes tag transfer without stimulating E2-ubiquitin thioester reactivity, in contrast with RING, SP-RING and some other E3 ligases. 
In summary, a unified model emerges for those E3 ligases that activate the reactivity of the thioester bond. The binding of an E3 enzyme restricts the conformations available for E2ubiquitin, which is then forced to adopt a configuration that optimally aligns the thioester for attack by the substrate's lysine. Future studies are required, however, to address how E3-E2ubiquitin complexes interact with their protein substrates.

\section{References}

1. Deshaies RJ, Joazeiro CA. Annu. Rev. Biochem. 2009; 78:399-434. [PubMed: 19489725]

2. Plechanovová A, Jaffray EG, Tatham MH, Naismith JH, Hay RT. Nature. 2012; 489:115-120. [PubMed: 22842904]

3. Dou H, Buetow L, Sibbet GJ, Cameron K, Huang DT. Nature Struct. Mol. Biol. 2012 http:// dx.doi.org/10.1038/nsmb.2379.

4. Pruneda JN, Stoll KE, Bolton LJ, Brzovic PS, Klevit RE. Biochemistry. 2011; 50:1624-1633. [PubMed: 21226485]

5. Pruneda JN, et al. Mol. Cell. 2012 http://dx.doi.org/10.1016/j.molcel.2012.07.001.

6. Wu PY, et al. EMBO J. 2003; 22:5241-5250. [PubMed: 14517261]

7. Yunus AA, Lima CD. Nature Struct. Mol. Biol. 2006; 13:491-499. [PubMed: 16732283]

8. Plechanovová A, et al. Nature Struct. Mol. Biol. 2011; 18:1052-1059. [PubMed: 21857666]

9. Reverter D, Lima CD. Nature. 2005; 435:687-692. [PubMed: 15931224]

10. Yunus AA, Lima CD. Mol. Cell. 2009; 35:669-682. [PubMed: 19748360]

11. Dou H, et al. Nature Struct. Mol. Biol. 2012; 19:184-192. [PubMed: 22266821]

12. Hamilton KS. Structure. 2001; 9:897-904. [PubMed: 11591345]

13. Wickliffe KE, Lorenz S, Wemmer DE, Kuriyan J, Rape M. Cell. 2011; 144:769-781. [PubMed: 21376237]

14. Saha A, Lewis S, Kleiger G, Kuhlman B, Deshaies RJ. Mol. Cell. 2011; 42:75-83. [PubMed: 21474069]

15. Kamadurai HB, et al. Mol. Cell. 2009; 36:1095-1102. [PubMed: 20064473] 


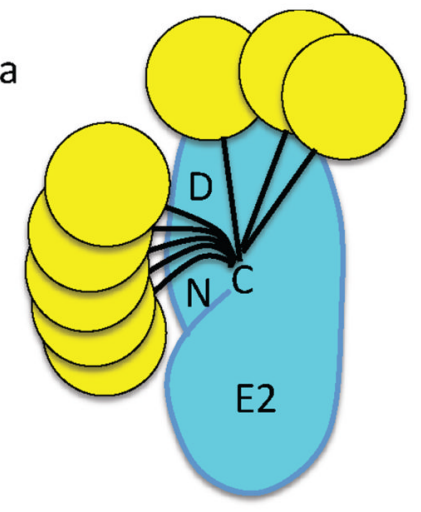

C

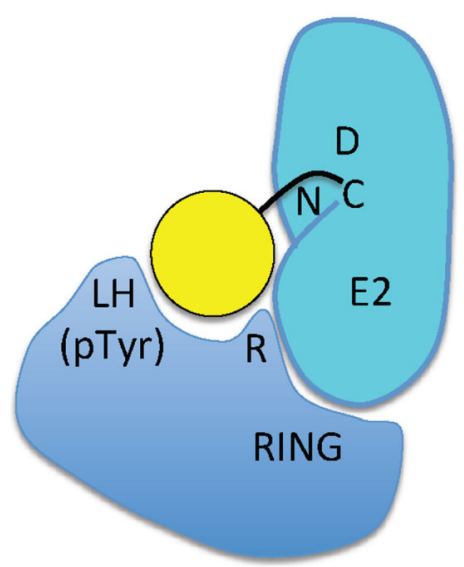

Cbl RING E3

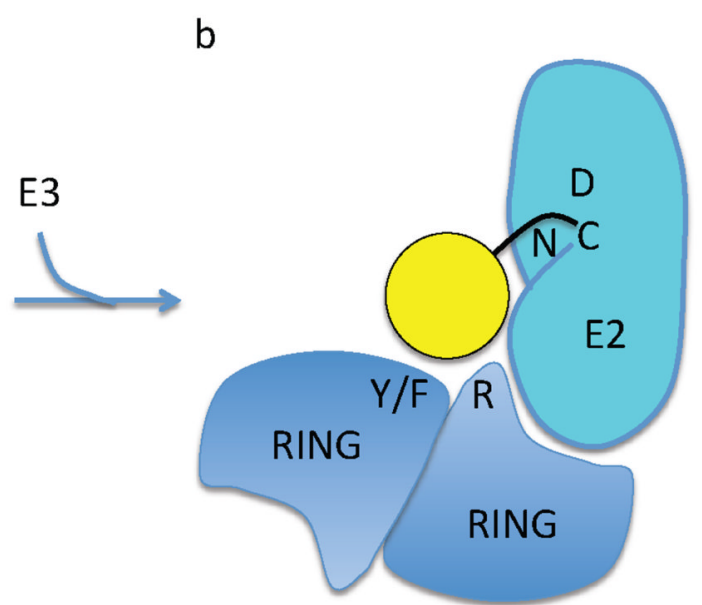

RNF4 and BIRC RING

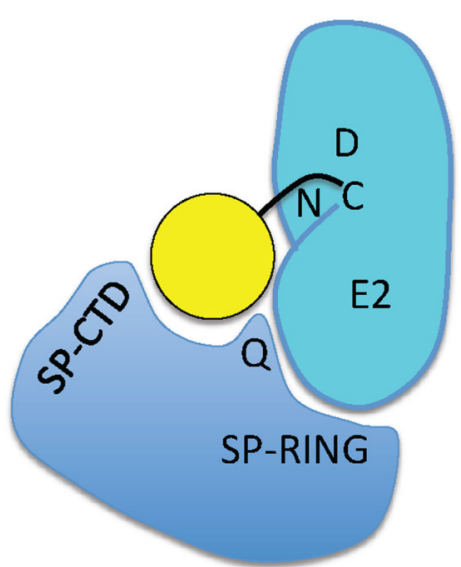

SP-RING E3

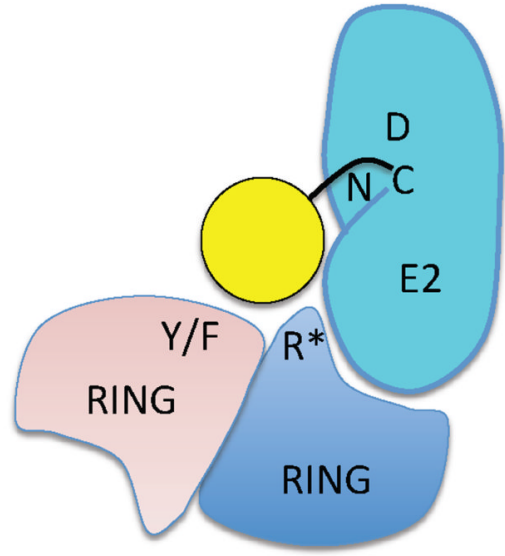

Homo-/Heterodimeric d

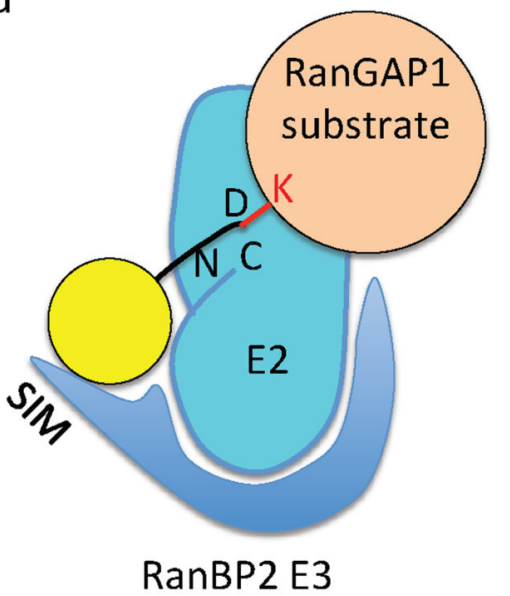

Figure 1. A unified model for ubiquitin transfer

The small protein ubiquitin is attached to E2 conjugating enzymes as an intermediate step before being transferred to other proteins through a process that is stimulated by E3 ligase enzymes. a, The transfer reaction is presumably hindered by a 'wobbling' movement of ubiquitin when attached to an isolated E2 protein. b, Plechanovová et al. ${ }^{2}$ and Dou et al. ${ }^{3}$ report crystal structures of E2-ubiquitin bound to dimeric E3 ligases of the RING family. They show that RING E3 ligases template E2-ubiquitin into an active conformation by establishing specific interactions with both ubiquitin and the E2 protein. In particular, an arginine and a tyrosine (or phenylalanine) of the E3 enzyme are crucial for securing ubiquitin into a position that activates transfer. As a result of these interactions, several residues in the $\mathrm{E} 2$ protein (such as an asparagine and an aspartate) are reorganized to facilitate the transfer reaction. c, Variations on this mechanism are used by monomeric RING, RING-like and some non-RING E3 ligases ${ }^{9-11}$ to activate transfer of ubiquitin (or ubiquitin-like proteins such as SUMO) from E2 proteins. 